\title{
Towards Improved User-Product Testing With Cognitively Enhanced Scenarios
}

\author{
Wilhelm Frederik van der Vegte \\ Delft University of Technology \\ Delft, The Netherlands
}

\author{
Niels C.C.M. Moes \\ Delft University of Technology \\ Delft, The Netherlands
}

\begin{abstract}
With the increasing information-intensiveness of products, users are challenged with expanding options and possible ways to interact. Rapidly escalating numbers of possible useroperation sequences hinder designers in anticipating all possible (unacceptable) outcomes. Interactively simulating product models with human subjects to explore all options is not practicable. Virtual simulation with computer models of users can open the way towards faster-than-real-time performance and investigation of massive numbers of interaction sequences. This paper reports on opportunities to improve realism of virtual-use simulations by incorporating knowledge about the workings of the human brain We elaborate how, in particular, cognitive-architecture simulations developed by cognitive scientists and error phenotypes identified in human reliability analysis (HRA) can extend a virtual-use simulation approach that we have proposed in foregoing work, by offering the prospective of generating interaction sequences with erroneous user actions unforeseen by the designer. We outline how such an integrated system can be implemented and also discuss validation issues.
\end{abstract}

\section{INTRODUCTION}

The rise of smart systems and ubiquitous computing has led to increasing information-intensiveness of products. As a consequence, issues that used to be associated with complex systems such as nuclear plants and airplanes are more and more becoming issues of everyday products such as cars, personal computers and even electric irons or microwave ovens. One of these issues is unforeseen use. The multitude of options that information-intensive products present to users can invoke 'erroneous' user actions governed by cognitive phenomena such as distraction, forgetfulness, information overload and learnability [1-4]

The growing complexity of possible interaction patterns can rapidly escalate the number of possible variations of user- operation sequences, and for designers it becomes increasingly difficult to foresee all possible outcomes, which might include unacceptable performance, failure, and even fatalities. To help solving this problem we aim to develop a method to predict possible outcomes of use during design, and thus allow anticipation before the product/system is realized. In order to deal with the large numbers of variations we have focused our efforts on computer simulations.

In previous work [5-7] we showed how complex interaction processes can be virtually tested by controlling conventional engineering simulations with use scenarios. Virtual testing allows early design evaluation since it can be done completely on a computer without deploying human subjects or physical prototypes. A scenario describes 'a way to use a product'. By unifying multiple ways of use in a timed hybrid automata (THA) representation, scenario bundles address the fact that most products are used in multiple ways, and also multiple times. In recent work we have reported how, specifically in the case of information-intensive products, simulations much faster than real time can be realized using a unified THA-based representation for all processes involved in using a product, including processes governed by the laws of physics [8]. The key advantage of simulations much faster than real time is that they allow large-scale exploration of the permutational variety of possible options.

However, a weakness of our approach in its present form is lack of realism of the user behaviors in the simulation. Currently user actions are 'programmed' into the scenario bundle (for instance, by taking the intended user manual as a starting point) based on conjecture by the designer, who can specify some additional variation by randomizing certain parameters. What we would like to simulate as well, however, is (the effects of) human behaviors beyond what the designer can think of - in particular, human 'errors' such as forgetting to check the status of the product when this is required, or taking too long to complete an action. By making simulations more realistic in this respect, we expect that designers can better 
anticipate and thus prevent user errors and/or their effects. After all, there is general agreement that primary responsibility to prevent human 'errors' is not with the end users themselves but with the designers of a system, since a design should inherently suit the capabilities of its users [e.g., 1,3].

Generally user 'errors' can be traced back to the workings of the human brain and the central nervous system, and the related human behaviors are studied by scientists in areas such as cognitive psychology, human motor science, human reliability analysis (HRA), and human-computer interaction (HCI). Some of their efforts have led to simulation algorithms or to formalizable knowledge that can otherwise be used in simulations. Most notably, cognitive architectures (CAs) such as ACT-R [9], EPIC [10], and SOAR [11], which have been developed by cognitive scientists, are interesting because they have been empirically validated and allow simulation of some of the key phenomena behind human error, such as learnability multitasking, learning, attention and distraction [12-14]. CAs are production-rule based blueprints of the operational structure of cognition. They predict the time the human brain needs for processing perceptual input and for directing motor operations. However they cannot predict the reasoning that determines which operation is performed in which situation, and this is why we still need scenario bundles. CA-based simulations start from a given task decomposition, i.e., an idealized sequence of operations as it can be extracted from an instruction manual.

In this paper we explore the opportunities to utilize CAs and related knowledge collected in associated areas of science with the objective to increase the realism of simulations involving virtual human users - in particular, but not exclusively, in combination with our approach that is briefly described in Section 3.

The paper is structured as follows: In the next section we discuss related achievements by others. Then, in Section 3 we briefly describe our approach of scenario-bundle based simulation (SBBS) without the proposed cognitive enhancements. Section 4 discusses the key cognitive aspects that have to be considered. In Section 5 we elaborate on our proposition to extend SBBS with cognitive abilities, and in Section 6 we wrap up with discussion and conclusions.

\section{RELATED WORK}

There have been few other efforts to operationalize CAs in simulations of the use of products. Since CAs originate from cooperation between computer scientists and psychologists [15], their application has long been limited to HCI [16]. With the emergence of ubiquitous technologies, everyday products are becoming increasingly information-intensive [17] and their use processes involve behaviors and interactions that are uncommon in HCI and have not been included in CAs. CAs offer effective shortcuts to simulate interactions with keyboards, monitors, etc. with disregard of physics [10]. Recently, similar specific solutions have been developed for driving cars [e.g., 18,19], and piloting aircraft [20] but so far no approach seems to exist that is widely applicable to information-intensive products and that has been devised for use in a design context. Possibly the only exception is the ACTR/DHM project at Mississippi State University, where researchers have attempted to enhance a 3D digital human model (DHM) with cognitive modules from ACT-R [21,22]. However, there are no reports on this project since 2009. At that time, the researchers had only considered modules for visually locating interaction elements, and had not considered further stages of human-product interaction.

\section{SIMULATING USE PROCESSES WITH TIMED HYBRID AUTOMATA: SBBS}

In [8] we presented a scenario-bundle based simulation (SBBS) approach to simulate the use of products based on a unified THA-based representation of actions and processes performed by - and taking place in - humans and artifacts. The idea behind this approach is to enable simulations much faster than real time by replacing the nowadays popular 3D productsimulation models for physics simulation with simple THA representations. 3D Simulation approaches, such as the ones based on which finite-element (FE) and multibody systems, are usually complex due to their dedication to accuracy, geometric completeness and, in the case of FE and other approaches based on spatial discretization, (approximation of) continuum physics, which requires keeping track of many points in space at many points in time. These simulations allow designers to investigate the dynamics of spatial changes in humans and products, and spatial manipulations that users are supposed to perform. However, in the case of information-intensive products, where successful use depends on information exchange and processing rather than on physics or human dexterity, such investigations are often not needed.

The logic of information exchange and processing can be described as discrete processes using THA. Advanced automata representations such as statecharts [23] can also be used to describe continuous behaviors based on (systems of) equations [e.g., 24]. Actually, the addition of continuous behaviors is what makes the automaton a hybrid one (i.e., the $\mathrm{H}$ in THA). This allows us to describe processes governed by physics - that is, up to a certain level of complexity, as long as keeping track of many points in space at many points in time is not needed.

As a consequence, we can simulate use processes based on

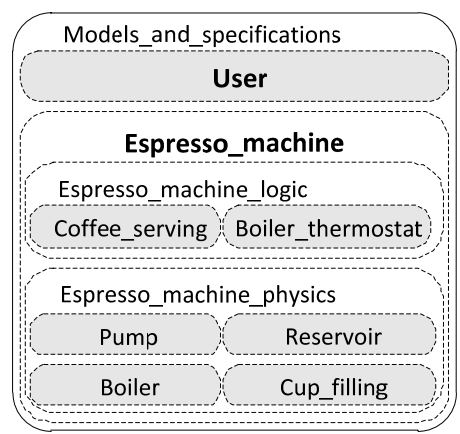

Figure 1.OVERVIEW OF SIMULATION CONSTRUCT FOR THE USE OF AN ESPRESSO MACHINE 


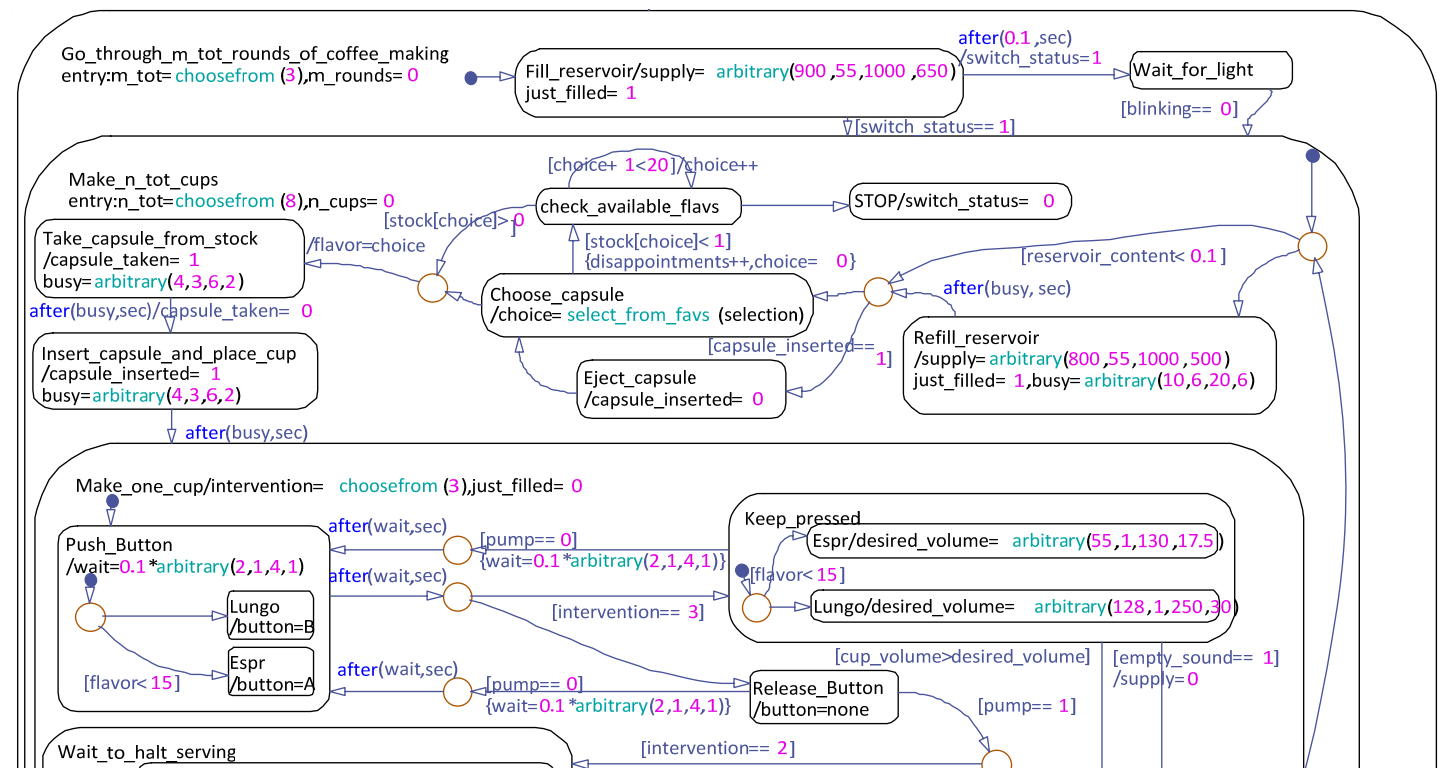

Figure 2. EXCERPT OF THE SCENARIO BUNDLE REPRESENTING ACTIONS BY A USER OPERATING AN ESPRESSO MACHINE ('USER' BLOCK IN FIG. 1).

models and specifications ${ }^{1}$ that are solely based on THA and that cover both the user and the product (as well as other involved artifacts and humans). As an example, Fig. 1 shows the models and specifications that we created with Matlab Simulink Stateflow to simulate the use of an espresso machine, They are uniformly represented as statecharts that are executed in parallel. Figure 2 shows an excerpt of the scenario bundle that describes actions by the virtual human user, which have been specified as reactions to supposedly perceived feedback from the espresso machine (e.g., a lamp blinks, the sound of the pump can be heard, etc.). The actions are represented as states (rounded rectangles) containing control commands and the perceived feedback is represented as transitions (arrows) to other actions, which take place based on specified conditions.

By executing this statechart together with similar models and specifications that describe the behavior of the espresso machine, simulations can be run at speeds up to $5,000 \times$ realtime, so that, for instance, a year of using the product can be simulated in less than two hours. An example of possible simulation output is shown in Fig. 3. Based on such output, the designer can, for instance, optimize the parameters related to the power-save mode in relation to targeted energy savings and assumed break-taking habits of the user. In the absence of functionality to predict durations of human actions, we built in custom functions to randomize durations within a given range, to be estimated by the designer. Similar functions are included to allow randomization of choices between different operation modes (e.g., lungo/large cup or espresso/small cup) and, for instance, filling level of the reservoir.

1 We distinguish physics models and logical specifications because physics models are typically based on validated relationships or derived from laws of nature, while logic is typically described based on assumptions or manmade program code

\section{CONSIDERATION OF COGNITIVE CAPABILITIES IN USE-PROCESS SIMULATION}

By means of scenario bundles, SBBS offers very limited support of cognitive activity related to human-product interaction. In that respect, a fully capable simulation approach should include the following three main aspects of cognition:

- Decision-making: Scenario bundles describe high-level decision-making but they disregard lower-level brain activity related to prioritizing and evaluating production
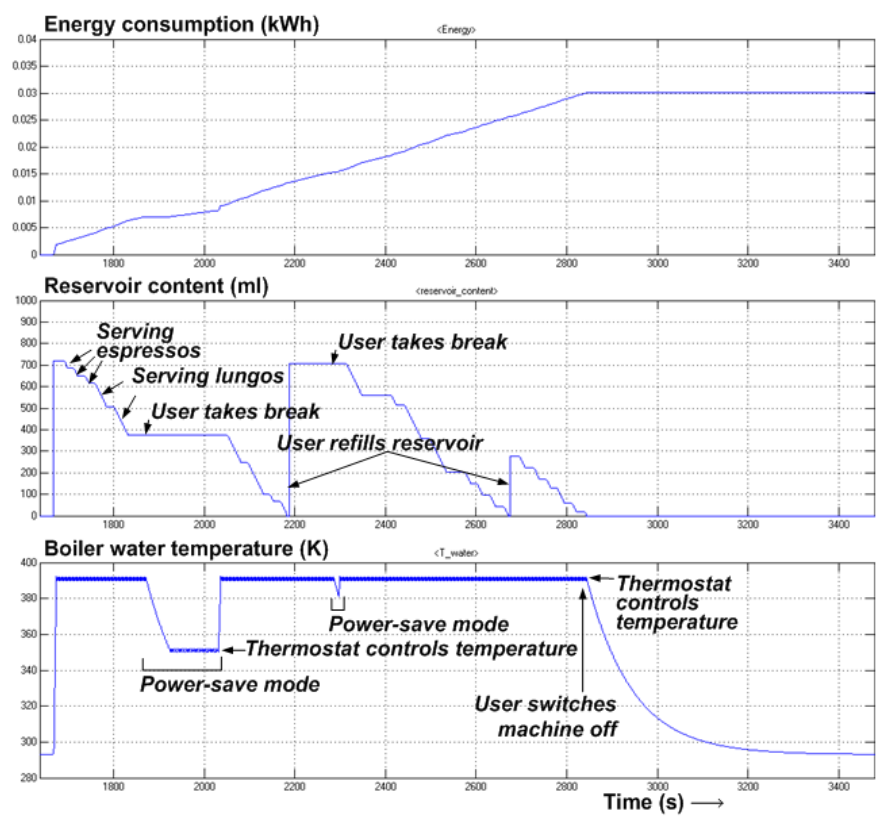

Figure 3. GRAPHICAL SIMULATION OUTPUT WITH EXPLANATORY TEXT 


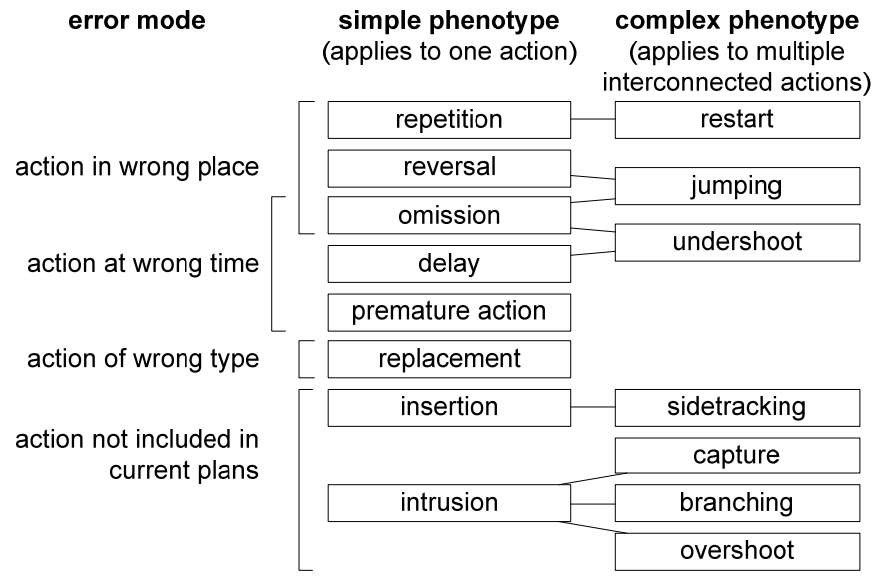

Figure 4. A TAXONOMY OF PHENOTYPES OF ERRONEOUS ACTIONS [25]

rules, accessing short-term/long-term memory, dividing attention, etc. Models of these activities belong to the core modules of CAs. Decision-making and the related cognitive processing are the source of the human 'errors' that we aim to reveal in simulations. In HRA, cognitive errors are considered at two levels: phenotype and genotype [25]. Error phenotypes (e.g., Fig. 4) can be observed in overt action, i.e., they correspond to aberrations at scenario level. However currently, a scenario bundle can only produce aberrations foreseen by its author. Genotype corresponds to the cognitive mechanisms causing errors and thus to CA-level processing. HRA and CA researchers [1,25-33], have presented genotype mechanisms and models, which are however not (yet) universally applicable in cognitive simulations.

- Motor control: in [7], we provisionally used unvalidated algorithms to simulate posture-to-posture transitions. Meanwhile, others introduced validated algorithms [3436]. These algorithms however exceed the functional capabilities that we currently need, since they offer full control of 3D spatial models. To extend capabilities of the HCI-centered motor models in CAs without considering full 3D control, Fitts's law [37] has been suggested [38]. In addition, recent refinements of Fitts's law and other invariants of motor control have been proposed [39-41], which can also be used to predict durations.

- Perception: regarding the cognitive aspects of perception, it is crucial to predict whether relevant events are detected, and if so, how much time is needed. In CAs, perception has been simplified by discretizing colors, zones of vision, etc. $[9,10]$.

\section{EXTENDING SBBS WITH COGNITIVE ABILITIES}

To extend scenario-bundle based simulations with cognitive abilities, we propose to link them to a cognitive architecture that intercepts the inputs to and outputs from the scenario bundle, and, based on computation of durations of cognitive processes, imposes delays (i) before the scenario

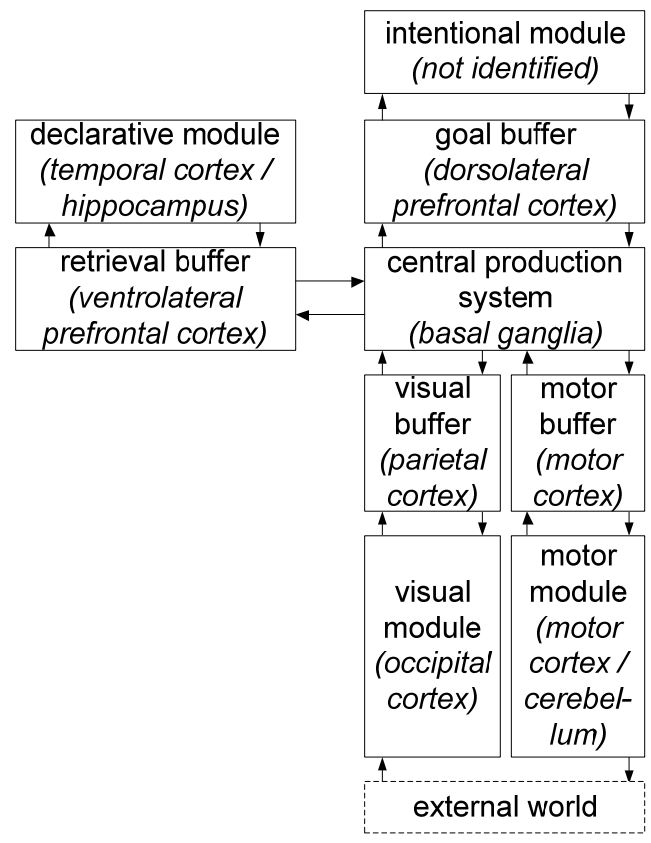

Figure 5 MODULES OF ACT-R (WITH ASSOCIATED REGIONS OF THE BRAIN, IN ITALICS) [9]

bundle can process its inputs from the artifact model and (ii) before it outputs the results of its processing to the artifact model. From the available CAs we propose to use ACT-R, since this architecture in particular has built-in perceptual and motor mechanisms that allow its models to interact with external simulations [18].

The architecture of ACT-R consists of four main modules, which are connected to a central production system through their respective buffers; most of these entities have been associated with particular regions of the brain (Fig. 5). The intentional or goal module keeps track of current goals and intentions, the declarative module retrieves information from memory, the visual module identifies objects in the visual field, and the motor module controls the limbs. The central production system coordinates in the behaviors of these modules. It can only respond to a limited amount of information that is deposited in the respective buffers of the modules by request of the central production system [9]. In ACT-R, user actions from a scenario appear as tasks, and scenario bundles combine these as structures of goals [cf. 42]. During simulation, the scenario proceeds from state to state based on fulfillment of conditions (user-perceived events). Thus, towards ACT-R the scenario acts as a new manifestation of the intentional module, feeding the goal buffer with upcoming tasks. Based on these, production rules control the motor module, which computes times for performing each successive task.

Novel extensions of ACT-R in the context of linking to SBBS concentrate on the motor module, adding currently missing models for operating everyday products based on motion invariants. The new models must (i) predict motor-task durations and (ii) prepare outputs compatible with simulations 


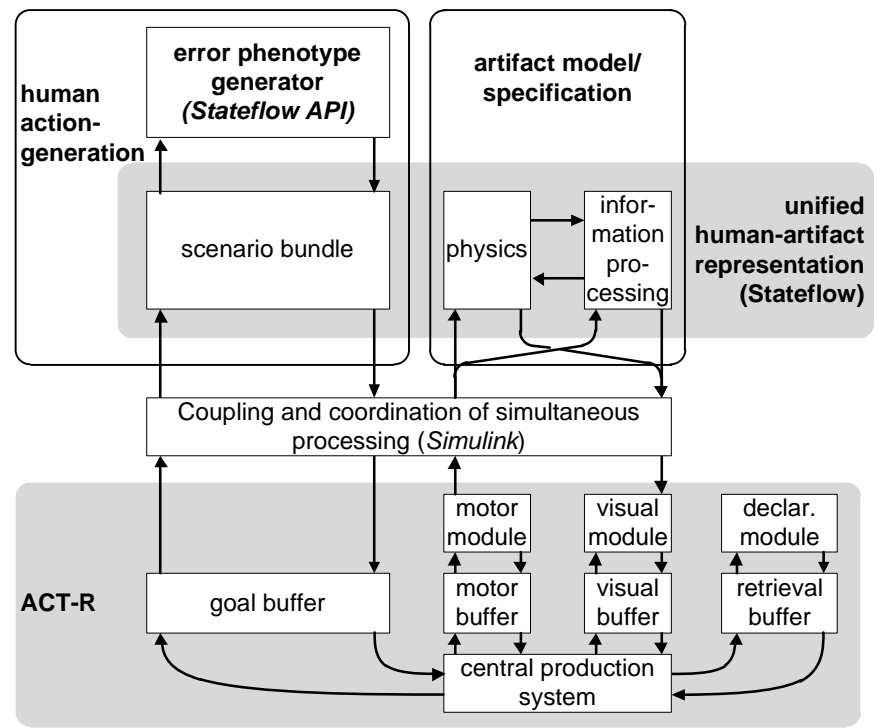

Figure 6. OVERVIEW OF THE INTENDED IMPLEMENTATION

of products and use environments. As is common practice for models that are newly added to CAs, they will have to be validated with human subjects.

From the error phenotypes in Fig. 4, the above combination of ACT-R and scenarios can only predict delays and, possibly, premature actions. One way to include more phenotypes is to (further) develop genotype models for ACTR's core modules based on [1,25-33]. Instead, we propose to explore and generate error phenotypes from scenarios. Manually assisted preparation of error phenotypes from a taxonomy by Reason [1] for ACT-R's inputs has been described in [43]. Hollnagel's taxonomy (Fig. 4) has the advantage over other taxonomies [e.g., 1,44] that it refers to the actions readily appearing in scenarios, which provides the opportunity to develop a novel module that generates errors automatically, producing deviations from the regular scenario by systematic reordering and reconnection. We propose to elaborate on this idea, aware of the following considerations:

- $\quad$ One group in Fig. 4 defines actions not regularly included. Additional actions foreseen in the scenario bundle can be explored but further meaningful actions will be hard to generate algorithmically.

- Exhaustive exploration of possible errors causes combinatorial explosion of the number of scenarios. Therefore, exploration should automatically stop at a given stage.

- We cannot directly assess probabilities of generated scenarios, which would be possible with validated genotypes. However, we aim to derive rules to predict probabilities of phenotypes from experiments involving human subjects.

Figure 6 gives an overview of the intended system. To connect its components in an initial prototype setup, we will use the simulation environment Matlab Simulink, which has proven capabilities to link CAs to statecharts (in Stateflow)
[19]. We expect that the error phenotype generator for alternative scenarios can be programmed using the Stateflow API.

\section{DISCUSSION AND CONCLUSIONS}

Above we have sketched a first outline of how we think that integrated simulation of use processes can be enhanced with cognitive capabilities. Realizing a system as we have proposed above is obviously a complex endeavor that requires further investigation. Therefore, we think that our first efforts should be aimed at validating the two basic issues: (i) is it feasible to identify a manageable collection of typical interactions that cover the use of most everyday products (outside HCI), and can the motor control related to these interactions be modeled as extensions of ACT-R's motor modules, and (ii) can a setup as is shown in Figure 6 predict the occurrence of real-life human errors? An interesting third question is, whether it is possible to derive rules concerning probability of typical error phenotypes.

We realize that even in the long run, validation for arbitrary information-intensive products is not a realistic objective. It probably means that if we can make the system work, it will have to prove itself in design practice. Another, related challenge that we have to deal with in future work is the evaluation of the effectiveness of the approach

In the above proposition we have focused on realizing fast simulations by largely ignoring 3D geometry and using simplified models of physics processes. We expect that such simulations will help designers to identify and anticipate possible human errors in the early stage of designing information-intensive products. Another interesting line of research would be to explore the possibilities of connecting similar cognitive simulation capabilities to 3D human models and artifact models. This may provide interesting insights in application areas where human dexterity is important, such as in sports or surgery.

\section{REFERENCES}

[1] Reason, J., 1990, Human error, Cambridge University Press, Cambridge.

[2] Norman, D.A., 1988, The psychology of everyday things, Basic Books, New York.

[3] Woods, D.D., Johannesen, L., Dekker, S., Cook, R. and Sarter, N., 1994, Behind human error, Ashgate Publishing, Ltd., Farnham.

[4] Wickens, C.D. and Hollands, J.G., 2000, Engineering psychology and human performance, Prentice-Hall, Upper Saddle River.

[5] Van der Vegte, W.F. and Horváth, I., 2012, "Theoretical underpinning and prototype implementation of scenario bundle-based logical control for simulation of human-artifact interaction", Computer-Aided Design (in press - doi: 10.1016/j.cad.2012.03.008).

[6] Van der Vegte, W.F. and Horváth, I., 2011, "Achieving Closed-Loop Control Simulation of Human-Artefact 
Interaction: A Comparative Review", Modelling and Simulation in Engineering, Vol. 2011, Article ID 675405.

[7] Van der Vegte, W.F., 2009, Testing virtual use with scenarios (PhD thesis), VSSD, Delft.

[8] van der Vegte, W.F. and Horváth, I., 2012, "Bulk simulation of using information-intensive products and product-service systems: formal underpinnings", Procs. TMCE, Karlsruhe, pp. 999-1012.

[9] Anderson, J.R., Bothell, D., Byrne, M.D. and Lebiere, C., 2004, "An integrated theory of the mind", Psychological Review, Vol. 111 (4), pp. 1036-1060.

[10] Kieras, D.E., Wood, S.D. and Meyer, D.E., 1997, "Predictive engineering models based on the EPIC architecture", ACM Transactions on Human-Computer Interaction, Vol. 4 (3), pp. 230-275.

[11] Laird, J.E., Newell, A. and Rosenbloom, P.S., 1987, "SOAR: an architecture for general intelligence", Artificial Intelligence, Vol. 33, pp. 1-64.

[12] Salvucci, D.D., Taatgen, N.A. and Borst, J.P., 2009, "Toward a unified theory of the multitasking continuum: From concurrent performance to task switching, interruption, and resumption", ACM, pp. 1819-1828.

[13] Taatgen, N., Van Rijn, H. and Anderson, J., 2007, "An integrated theory of prospective time interval estimation: The role of cognition, attention, and learning", Psychological Review, Vol. 114 (3), pp. 577598.

[14] Salvucci, D.D., Zuber, M., Beregovaia, E. and Markley, D., 2005, "Distract-R: Rapid prototyping and evaluation of in-vehicle interfaces", Procs. CHI, ACM, pp. 581-589.

[15] Newell, A. and Card, S., 1985, "The prospects for psychological science in human-computer interaction", Human-Computer Interaction, Vol. 1 (3), pp. 209-242.

[16] Byrne, M.D., 2005, "Cognitive architectures in HCI: Present work and future directions", Procs. Int. Conf. on Human Computer Interaction, Las Vegas.

[17] Poslad, S., 2009, Ubiquitous computing: smart devices, environments and interactions, John Wiley \& Sons Inc.

[18] Salvucci, D.D., 2006, "Modeling driver behavior in a cognitive architecture", Human Factors: The Journal of the Human Factors and Ergonomics Society, Vol. 48 (2), p. 362.

[19] Weber, L., Baumann, M., Lüdtke, A. and Steenken, R., 2009, "Modellierung von Entscheidungen beim Einfädeln auf die Autobahn (Modelling decisions when merging onto the highway)", in: Lichtenstein, A., Stößel, C., Clemens, C. (Eds.), Der Mensch im Mittelpunkt technischer Systeme, 8. Berliner Werkstatt, Mensch-Maschine-Systeme, Vol. 22, VDI-Verlag, Düsseldorf, pp. 27-28.

[20] Lüdtke, A. and Osterloh, J., 2009, "Simulating Perceptive Processes of Pilots to Support System
Design", Lecture Notes in Computer Science, Vol. 5726, pp. 471-484.

[21] Carruth, D.W., Thomas, M.D., Robbins, B. and Morais, A., 2007, "Integrating perception, cognition and action for digital human modellling", Lecture Notes in Computer Science (4561), pp. 333-342.

[22] Robbins, B., Carruth, D. and Morais, A., 2009, "Bridging the gap between HCI and DHM: the modeling of spatial awareness within a cognitive architecture", Lecture Notes in Computer Science, Vol. 5620, pp. 295-304.

[23] Harel, D., 1987, "Statecharts: a visual formalism for complex systems", Science of Computer Programming, Vol. 8, pp. 231-274.

[24] Mathworks, 2011, Stateflow for state diagram modeling: user's guide, The MathWorks, Inc., Natick.

[25] Hollnagel, E., 1993, Human reliability analysis: Context and control, Academic Press, London.

[26] Endsley, M.R., 1999, "Situation awareness in aviation systems", Handbook of aviation human factors, pp. 257-276.

[27] Norman, D.A., 1983, "Design rules based on analyses of human error", Communications of the ACM, Vol. 26 (4), pp. 254-258.

[28] Orasanu, J. and Martin, L., 1998, "Errors in aviation decision making: A factor in accidents and incidents", Procs. HESSD, Seattle, pp. 100-107.

[29] Rasmussen, J., 1986, Information processing and human-machine interaction, North-Holland, New York.

[30] Shorrock, S.T. and Kirwan, B., 2002, "Development and application of a human error identification tool for air traffic control", Applied Ergonomics, Vol. 33 (4), pp. 319-336.

[31] Byrne, M.D. and Bovair, S., 1997, "A working memory model of a common procedural error", Cognitive science, Vol. 21 (1), pp. 31-61.

[32] Gray, W.D., 2000, "The nature and processing of errors in interactive behavior", Cognitive science, Vol. 24 (2), pp. 205-248.

[33] Trafton, J.G., Altmann, E.M. and Ratwani, R.M., 2011, "A memory for goals model of sequence errors", Cognitive Systems Research, Vol. 12 (2), pp. 134-143.

[34] Singh, B., Hicks, B., Medland, A.J., Mullineux, G. and Molenbroek, J.M., 2010, "A contraint based human model for simulating and predicting postures", Procs. TMCE, Vol. 1, Ancona, pp. 221-229.

[35] Abdel-Malek, K., Yang, J.Z., Kim, J.H., Marler, T., Beck, S., Swan, C., Frey-Law, L., Mathai, A., Murphy, C., Rahmatallah, S. and Arora, J., 2007, "Development of the virtual-human santos (TM)", Digital Human Modeling, Vol. 4561, pp. 490-499.

[36] Park, W., Chaffin, D.B., Martin, B.J. and Yoon, J., 2008, "Memory-based human motion simulation for computer-aided ergonomic design", IEEE 
Transactions on Systems Man and Cybernetics Part ASystems and Humans, Vol. 38 (3), pp. 513-527.

[37] Fitts, P.M., 1951, Human engineering for an effective air navigation and traffic control system, National Research Council, Washington D.C.

[38] Jax, S.A., Rosenbaum, D.A., Vaughan, J. and Meulenbroek, R.G.J., 2003, "Computational motor control and human factors: modeling movements in real and possible environments", Human Factors, Vol. 45 (1), pp. 5-27.

[39] Beamish, D., Bhatti, S., Chubbs, C.S., MacKenzie, I.S., Wu, J. and Jing, Z., 2009, "Estimation of psychomotor delay from the Fitts' law coefficients", Biological cybernetics, Vol. 101 (4), pp. 279-296.

[40] Bullock, D. and Grossberg, S., 1988, "Neural dynamics of planned arm movements: emergent invariants and speed-accuracy properties during trajectory formation", Psychological Review, Vol. 95 (1), pp. 49-90.

[41] Guigon, E., Baraduc, P. and Desmurget, M., 2007, "Computational motor control: redundancy and invariance", Journal of neurophysiology, Vol. 97 (1), pp. 331-347.

[42] Taatgen, N. and Lee, F., 2003, "Production compilation: A simple mechanism to model complex skill acquisition", Human Factors: The Journal of the Human Factors and Ergonomics Society, Vol. 45 (1), pp. 61-76.

[43] Begosso, L.C. and Filgueiras, L.V.L., 2006, "Human error simulation as an aid to HCI design for critical systems", Procs. IHC, ACM, Rio Grande do Norte, pp. 120-127.

[44] Embrey, D., 1986, "SHERPA: A systematic human error reduction and prediction approach", Int. Meeting on Advances in Nuclear Power Systems, Knoxville, 1986. 